\title{
Comparison of Spinous Process-Splitting Laminectomy versus Conventional Laminectomy for Lumbar Spinal Stenosis
}

\author{
Masashi Uehara ${ }^{1}$, Jun Takahashi ${ }^{2}$, Hiroyuki Hashidate ${ }^{3}$, Keijiro Mukaiyama ${ }^{2}$, \\ Shugo Kuraishi ${ }^{2}$, Masayuki Shimizu ${ }^{2}$, Shota Ikegami ${ }^{2}$, Toshimasa Futatsugi ${ }^{2}$, \\ Nobuhide Ogihara ${ }^{4}$, Hiroki Hirabayashi ${ }^{5}$, Hiroyuki Kato ${ }^{2}$ \\ ${ }^{I}$ Department of Orthopaedic Surgery, Yodakubo Hospital, Nagawa-machi, Japan \\ ${ }^{2}$ Department of Orthopaedic Surgery, Shinshu University School of Medicine, Matsumoto, Japan \\ ${ }^{3}$ Department of Orthopaedic Surgery, Shinonoi General Hospital, Nagano, Japan \\ ${ }^{4}$ Department of Orthopaedic Surgery, Ina Central Hospital, Ina, Japan \\ ${ }^{5}$ Department of Orthopaedic Surgery, Marunouchi Hospital, Matsumoto, Japan
}

Study Design: Seventy-five patients who had been treated for lumbar spinal stenosis (LSS) were reviewed retrospectively.

Purpose: Invasion into the paravertebral muscle can cause major problems after laminectomy for LSS. To address these problems, we performed spinous process-splitting laminectomy. We present a comparative study of decompression of LSS using 2 approaches.

Overview of Literature: There are no other study has investigated the lumbar spinal instability after spinous process-splitting laminectomy.

Methods: This study included 75 patients who underwent laminectomy for the treatment of LSS and who were observed through follow-ups for more than 2 years. Fifty-five patients underwent spinous process-splitting laminectomy (splitting group) and 20 patients underwent conventional laminectomy (conventional group). We evaluated the clinical and radiographic results of each surgical procedure.

Results: Japanese Orthopaedic Association score improved significantly in both groups two years postoperatively. The following values were all significantly lower, as shown with $p$-values, in the splitting group compared to the conventional group: average operating time ( $p=0.002)$, postoperative $C$-reactive protein level $(p=0.006)$, the mean postoperative number of days until returning to normal body temperature $(p=0.047)$, and the mean change in angulation 2 years postoperatively $(p=0.007)$. The adjacent segment degeneration occurred in 6 patients $(10.9 \%)$ in the splitting group and 11 patients $(55.0 \%)$ in the conventional group.

Conclusions: In this study, the spinous process-splitting laminectomy was shown to be less invasive and more stable for patients with LSS, compared to the conventional laminectomy.

Keywords: Lumbar spinal stenosis; Spinous process-splitting laminectomy; Postoperative low back pain; Paravertebral muscle, posterior approach

Received Dec 30, 2013; Revised Jan 29, 2014; Accepted Feb 19, 2014

Corresponding author: Jun Takahashi

Department of Orthopaedic Surgery, Shinshu University School of Medicine,

3-1-1 Asahi, Matsumoto 390-8621, Japan

Tel: +81-263-37-2659, Fax: +81-263-35-8844, E-mail:jtaka@shinshu-u.ac.jp 


\section{Introduction}

Laminectomy is widely performed in treating lumbar spinal stenosis (LSS). However, approach-related morbidity resulting from iatrogenic soft tissue injury has become a major problem, which includes the paraspinal muscle injury due to its extensive detachment from the posterior aspect of the lumbar spine $[1,2]$. Watanabe et al. [3] reported that in lumbar spinous process-splitting laminectomy, the muscular attachment is left intact and this decreases the degree of postoperative paraspinal muscular atrophy. In this procedure, a wide visualization of the central canal and the lateral recess can be obtained by a bilateral retraction of the split spinous processes and the attached ligaments, allowing trouble-free access to the nerves. The preserved midline osteoligamentous structures occasionally limit access to the nerve tissues, disturbing the visualization and decompression of the nerve roots in fenestration and laminotomy. However, relatively few studies have reported the clinical effects in the postoperative period, from the paraspinal preservation by lumbar spinous process-splitting laminectomy; and no other study has investigated the lumbar spinal instability after this procedure. In this report, we present a retrospective comparative study on decompression of LSS using both conventional and spinous process-splitting approaches.

\section{Materials and Methods}

This study was approved by the Investigational Review Board of Shinshu University hospital. We included 75 patients (43 men, 32 women; mean age, $71.3 \pm 9.2$ years; range, 40-89 years) who underwent laminectomy (less than 2 intervertebral levels) without fixation for the treatment of LSS and who were observed for more than 2 years or until revision surgery, between October 2003 to January 2011. Fifty-five patients (33 men, 22 women; mean age, $71.0 \pm 9.3$ years; range, $40-85$ years) underwent spinous process-splitting laminectomy (splitting group) and 20 patients ( 10 men, 10 women; mean age, $72.2 \pm 9.1$ years; range, 57-89 years) underwent conventional laminectomy (conventional group). These surgical treatments were indicated for the patients with cauda equina syndrome or radiculopathy caused by cauda equina or nerve root compression without lumbar instability. We performed spinal fusion surgery in the following cases: change in angulation $>10^{\circ}$, change in translation $>3 \mathrm{~mm}$ between flexion and extension, posterior spreading $>5^{\circ}$ in flexion, and existence of low back pain with motion.

\section{Operative technique for spinous process-splitting laminectomy}

Here, we describe a case of single-level (L4-5) decompression, according to the surgical method described by Watanabe et al. [3]. A posterior midline skin incision was made between the L4 and L5 spinous processes. Soft tissue was dissected up to the tip of the L4 and L5 spinous processes using an electric knife. The cortex at the tip of the L4 and L5 spinous processes was removed at the midline by approximately $2 \mathrm{~cm}$ using a $4-\mathrm{mm}$ diamondtipped bur. The spinous process was divided at the base, detached from the lamina using a cob elevator, and retracted using TrimLine (Medtronic, Sofamor Danek, Memphis, TN, USA). The remaining spinous process was removed using Luer bone rongeur forceps. Then, we performed laminectomy. After decompression, suction drain was placed in the epidural space. After that, the spinous process and interspinal ligament, which were split, were sutured by absorbable threads. After the surgery, walking was allowed on the same day and the lumbar corset was not used (Figs. 1-3).

\section{Operative technique for conventional laminectomy}

A posterior midline skin incision was made between the L4 and L5 spinous processes. The paravertebral muscles from the spinous processes to the medial facet joints were cut using an electric knife. The L4 and cranial half of the L5 spinous processes were excised. Laminectomy was performed using an air drill at the yellow ligament attachment site of the L4 lamina. Medial half facetectomy was performed, and the right and left L5 nerve roots were decompressed. After decompression, a suction drain was placed in the epidural space (Fig. 4). The post-surgical management is the same as in spinous process-splitting laminectomy.

\section{Evaluation}

The Japanese Orthopaedic Association (JOA) scoring system for lumbar spinal disorders (full score, 29 points) [4] was used to evaluate the clinical results preoperatively 

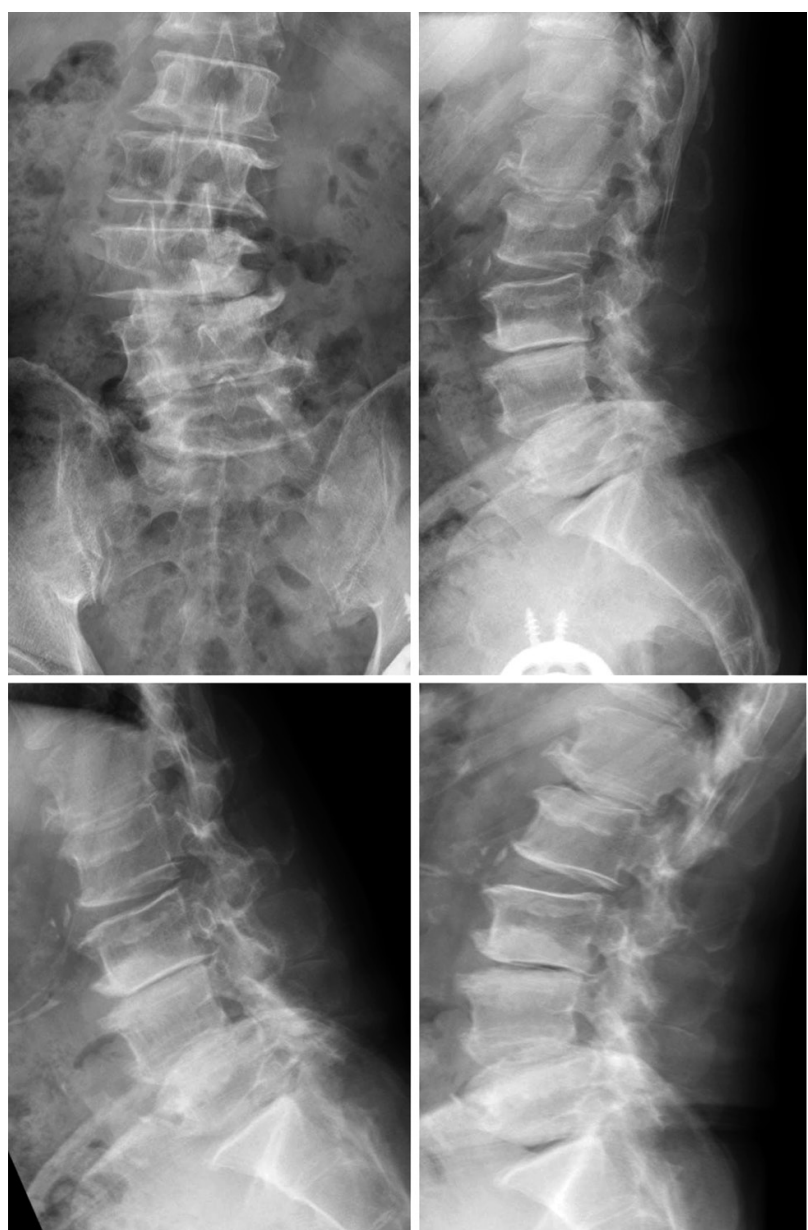

Fig. 1. Preoperative radiographs.
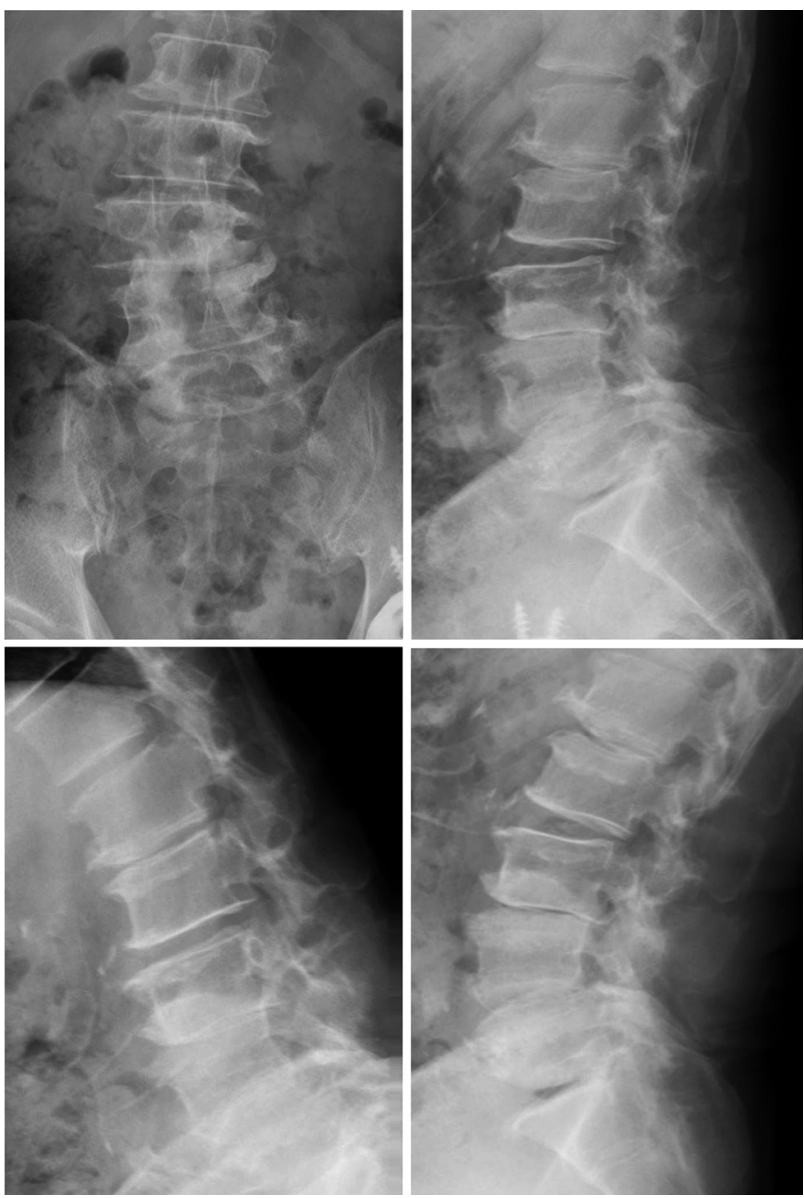

Fig. 2. Radiographs 2 years after the surgery. Spinous process-splitting laminectomy from $\mathrm{L} 3$ to $\mathrm{L} 5$ was performed. There was no spinal instability 2 years after the surgery. The Japanese Orthopaedic Association score improved from 9 to 22 points.
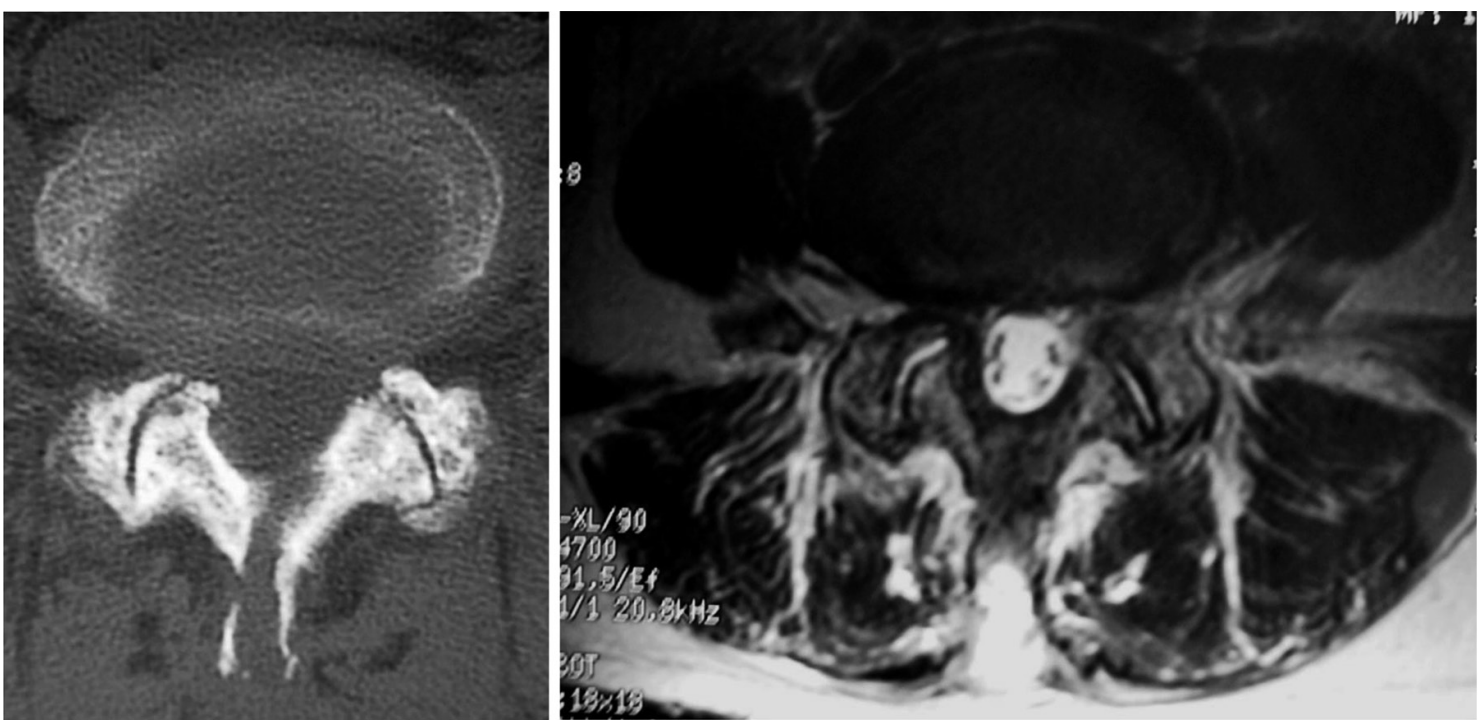

Fig. 3. Postoperative computed tomography and magnetic resonance images of a patient in the splitting group. The spinous process was split and sutured, and the paravertebral muscle showed little damage at 3 months after the surgery. 
Table 1. Characteristics of the study population

\begin{tabular}{lccc} 
Characteristic & Splitting group $(\mathrm{n}=55)$ & Conventional group $(\mathrm{n}=20)$ & $p$-value \\
\hline Mean age (yr) & $71.0 \pm 9.3$ & $72.2 \pm 9.1$ & 0.801 \\
\hline Decompression level (intervertebral level) & $1.5 \pm 0.5$ & $1.8 \pm 0.4$ & 0.112 \\
Surgical time (min) & $82.3 \pm 32.7$ & $121.2 \pm 52.6$ & 0.002 \\
\hline Intraoperative blood loss $(\mathrm{g})$ & $77.4 \pm 99.0$ & $206 \pm 233$ & 0.059 \\
\hline Postoperative blood loss $(\mathrm{g})$ & $414 \pm 396$ & $439 \pm 255$ & 0.323 \\
\hline
\end{tabular}

Values are presented as mean \pm standard deviation.

a) Determined by the Mann-Whitney U test.

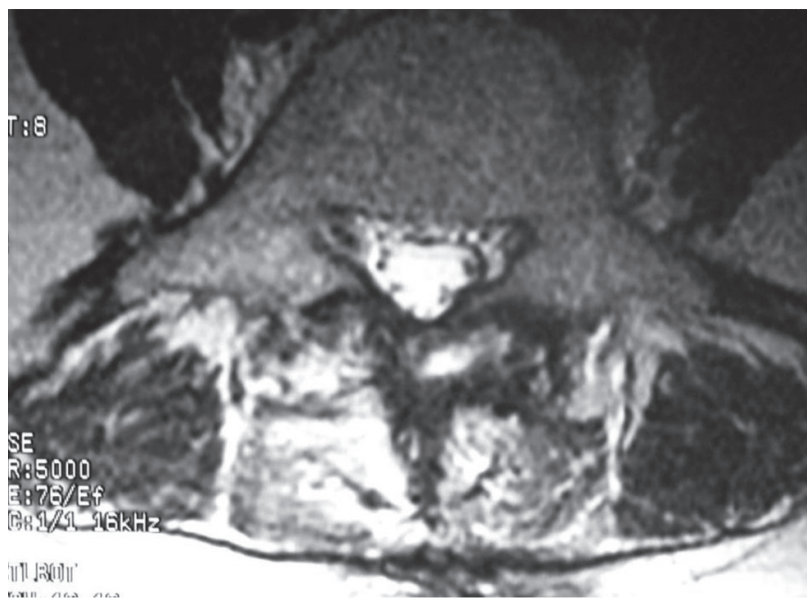

Fig. 4. Postoperative magnetic resonance image of a patient in the conventional group. The spinous process was excised and high signal change was observed in the paravertebral muscle, 3 months after surgery.

and 2 years postoperatively. The recovery rate was calculated using the method described by Hirabayashi et al. [5], which compares preoperative and postoperative JOA scores according to the following equation: recovery rate $(\%)=($ postoperative score-preoperative score $) \times 100 /($ full score-preoperative score). The occurrences of surgical complications, operating time, intra- and postoperative blood loss volume, length of hospital stay, C-reactive protein (CRP) level on the first postoperative day, and creatine kinase (CK) level on the first postoperative day were also investigated.

Translation distance of the vertebra at the most unstable intervertebral level was calculated using preoperative and 2-year postoperative flexion and extension radiographs. The difference in slip angle between maximum flexion and maximum extension at the most unstable intervertebral level was also measured. Radiographic evidence of adjacent segment degeneration
(ASD) was evaluated at the 2-year follow-up, and it was determined using pre-established criteria as follows: new anterior osteophyte formation or enlargement of existing osteophytes, increased or new narrowing of the disc space ( $>30 \%)$, new or increased calcification of the anterior longitudinal ligament, and formation of radial osteophytes [6]. Radiographic measurements were obtained by the first author, who was not involved in the surgeries.

We evaluated the postoperative JOA scores and radiographic data at 2 years postoperatively or just before the revision surgery. Data were analyzed by using the Wilcoxon signed-rank test for continuous data and the MannWhitney $\mathrm{U}$ test for categorical data using SPSS software (SPSS Japan Inc., an IBM company, Tokyo, Japan). The level of statistical significance was set at $p<0.05$.

\section{Results}

The patients' follow-up periods ranged from 4 to 83 months (mean, 25.6 \pm 23.3 months). The mean number of laminectomy levels was 1.6 intervertebral levels (range, 1-2 levels; 1 level in 30 patients, 2 levels in 45 patients). The mean number of laminectomy levels in the splitting group was 1.5 intervertebral levels (range, 1-2 levels; 1 level in 25 patients, 2 levels in 30 patients). The mean number of laminectomy levels in the conventional group was 1.8 intervertebral levels (range, 1-2 levels; 1 level in 5 patients, 2 levels in 15 patients) (Table 1).

\section{Clinical results}

The operating time and intra- and postoperative blood loss are summarized in Table 1 . The average operating time was $82.3 \pm 32.7$ minutes (range, $40-189$ minutes) in the splitting group and 121.2 \pm 52.6 minutes (range, 
48-231 minutes) in the conventional group. The average operating time in the splitting group was significantly shorter than the conventional group $(p=0.002)$. The average intraoperative blood loss was $77 \pm 99 \mathrm{~mL}$ (range, $10-650 \mathrm{~mL}$ ) in the splitting group and $206 \pm 234 \mathrm{~mL}$ (range, $10-800 \mathrm{~mL}$ ) in the conventional group, showing no significant difference between them $(p=0.059)$. The average postoperative blood loss was $414 \pm 396 \mathrm{~mL}$ (range, $60-2,460 \mathrm{~mL}$ ) in the splitting group and $439 \pm 255 \mathrm{~mL}$ (range, 140-970 mL) in the conventional group, showing no significant difference between them $(p=0.323)$. A total of 5 cases of complications were reported, including hematoma requiring revision surgery in 1 patient from the splitting group, deep vein thrombosis in $1 \mathrm{pa}$ tient from the splitting group, and surgical site infection in 2 patients from the splitting group and in 1 patient from the conventional group. In the splitting group, the mean preoperative and 2-year postoperative JOA scores were $12.8 \pm 3.6$ points (range, $7-20$ ) and $22.6 \pm 6.0$ points (range, 5-29), respectively. The mean score improved significantly $(p<0.001)$, as analyzed by the Wilcoxon signed-rank test. In the conventional group, the mean preoperative and 2-year postoperative JOA scores were
$14.3 \pm 5.3$ points (range, $8-24$ points) and $20.4 \pm 7.2$ points (range, $6-28$ points), respectively. In both groups, the mean score improved significantly $(p=0.021)$ (Fig. $5)$. The recovery rate at 2 years postoperatively was $63.8 \% \pm 28.4 \%$ (range, $-20 \%$ to $100 \%$ ) in the splitting group and $40.3 \% \pm 42.9 \%$ (range, $-40 \%$ to $83.3 \%$ ) in the conventional group, showing no significant difference between the groups ( $p=0.113$ ). In the splitting group, the JOA score for low back pain improved significantly from $1.9 \pm 0.6$ points (range, $1-3$ points) preoperatively to $2.4 \pm 0.6$ points (range, $1-3$ points) 2 years postoperatively $(p=0.008)$. In the conventional group, the JOA score for low back pain improved from $1.7 \pm 0.5$ points (range, $1-2$ points) preoperatively to $2.3 \pm 0.8$ points (range, 1-3 points) 2 years postoperatively, showing no significant difference between the groups $(p=0.414)$. The JOA scores for low back pain 2 years postoperatively were not significantly different between the two groups ( $p=0.572$ ).

The mean number of days until the first postoperative walk was $2.2 \pm 1.4$ days in the splitting group and $2.7 \pm 1.8$ days in the conventional group, showing no significant difference between the groups $(p=0.193)$. The

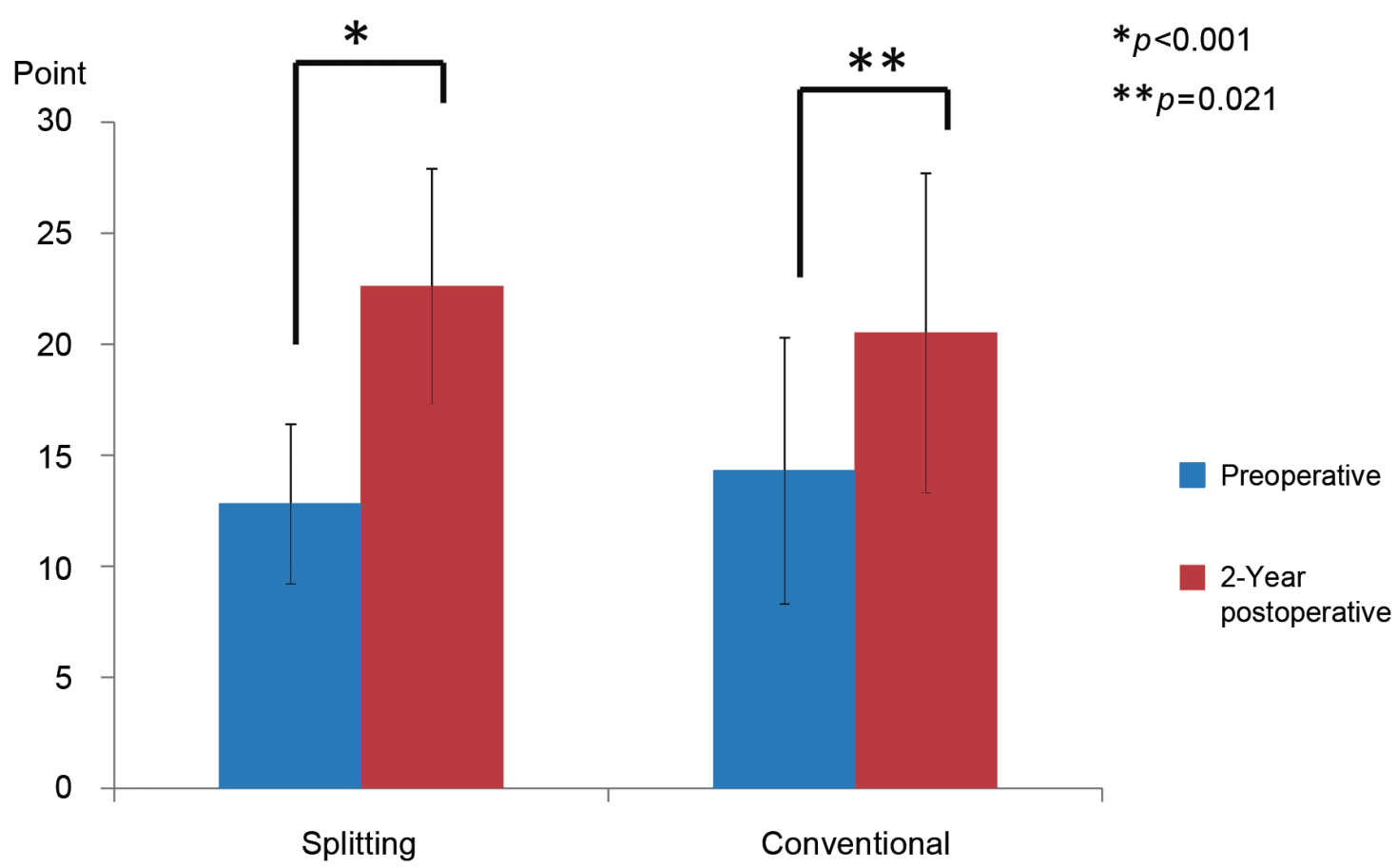

Fig. 5. Preoperativ and postoperative Japanese Orthopaedic Association (JOA) scores. In the splitting group, the mean preoperative and 2-year postoperative JOA scores were 12.8 points and 22.6 points, respectively; the score improved significantly. In the conventional group, the mean preoperative and 2-year postoperative JOA scores were 14.3 points and 20.5 points, respectively; the score improved significantly. 
Table 2. Comparison of clinical results

\begin{tabular}{lccc} 
Characteristic & Splitting group $(\mathrm{n}=55)$ & Conventional group (n=20) & $p$-value \\
CRP on first postoperative day $(\mathrm{mg} / \mathrm{dL})$ & $0.68 \pm 0.64$ & $1.8 \pm 3.6$ & 0.006 \\
CK on first postoperative day $(\mathrm{mg} / \mathrm{dL})$ & $176 \pm 104$ & $352 \pm 566$ & 0.161 \\
\hline First postoperative walk (day) & $2.2 \pm 1.4$ & $2.7 \pm 1.8$ & 0.193 \\
\hline Return to normal temperature (day) & $2.7 \pm 1.6$ & $3.6 \pm 1.9$ & 0.047 \\
\hline Length of hospital stay (day) & $17.6 \pm 4.5$ & $20.1 \pm 6.2$ & 0.100 \\
\hline Recovery rate of JOA score (\%) & $63.8 \pm 28.4$ & $40.3 \pm 42.9$ & 0.113 \\
Change in translation (mm) & $0.25 \pm 1.06$ & $0.71 \pm 1.53$ & 0.139 \\
\hline Change in angulation (degree) & $0.7 \pm 2.2$ & $3.5 \pm 3.7$ & 0.007 \\
\hline
\end{tabular}

Values are presented as mean \pm standard deviation.

CRP, C-reactive protein; CK, creatine kinase; JOA, Japanese Orthopaedic Association.

a) Determined by the Mann-Whitney U test.

mean number of days until returning to normal body temperature $\left(<37^{\circ} \mathrm{C}\right)$ postoperatively was $2.7 \pm 1.6$ days in the splitting group and $3.6 \pm 1.9$ days in the conventional group, and this was significantly lower in the splitting group than in the conventional group $(p=0.047)$. The average duration of hospital stay was $17.6 \pm 4.6$ days in the splitting group and $20.2 \pm 6.2$ days in the conventional group, which was not significantly different $(p=0.100)$. The mean CRP and CK levels on the first postoperative day were $0.68 \pm 0.64 \mathrm{mg} / \mathrm{dL}$ and $176 \pm 104 \mathrm{mg} / \mathrm{dL}$ in the splitting group and $1.7 \pm 3.6 \mathrm{mg} / \mathrm{dL}$ and $352 \pm 566 \mathrm{mg} / \mathrm{dL}$ in the conventional group, respectively. The CRP levels were significantly greater in the conventional group than in the splitting group $(p=0.006)$. The CK levels were not significantly different $(p=0.161)$. The comparisons of the clinical results between the two groups are summarized in Table 2.

\section{Radiographic results}

The mean translation distance increased significantly from $0.29 \pm 0.68 \mathrm{~mm}$ preoperatively to $0.55 \pm 1.4 \mathrm{~mm} 2$ years postoperatively in the splitting group $(p=0.011)$. The mean translation distance increased from $0.12 \pm 0.49 \mathrm{~mm}$ preoperatively to $0.78 \pm 1.9 \mathrm{~mm} 2$ years postoperatively in the conventional group, which was not significant $(p=0.068)$. The mean change in angulation between flexion and extension increased significantly from $1.9^{\circ} \pm 2.6^{\circ}$ preoperatively to $2.6^{\circ} \pm 3.6^{\circ} 2$ years postoperatively in the splitting group ( $p=0.068$ ). The mean change in angulation between flexion and extension increased significantly from $2.4^{\circ} \pm 3.6^{\circ}$ preoperatively to $5.8^{\circ} \pm 5.3^{\circ} 2$ years postoperatively in the conventional group ( $p=0.004)$. The changes in translation from before surgery to 2 years after surgery was $0.25 \pm 1.1 \mathrm{~mm}$ in the splitting group and $0.71 \pm 1.5$ $\mathrm{mm}$ in the conventional group. This was not significantly different between the two groups ( $p=0.139$ ). The changes in angulation from before surgery to 2 years after surgery were $0.67^{\circ} \pm 2.2^{\circ}$ in the splitting group and $3.5^{\circ} \pm 3.7^{\circ}$ in the conventional group. This was significantly lower in the splitting group than in the conventional group ( $p=0.007$ ). ASD occurred in 6 patients (10.9\%) in the splitting group and 11 patients $(55.0 \%)$ in the conventional group. This was significantly lower in the splitting group than in the conventional group $(p<0.001)$.

\section{Revision surgery}

Revision surgeries were performed for 9 patients: 7 $(12.7 \%)$ in the splitting group and $2(10.0 \%)$ in the conventional group. The mean period of time from first surgery to revision surgery was 18.3 months (range, 4-34 months). The reasons for revision surgery and their occurrences in patients were as follows: slip progression in 2 patients, radiculopathy at the intervertebral foramen in 3 patients, restenosis in 1 patient, adjacent vertebral fracture in 1 patient, and disc herniation in 2 patients. One patient $(1.8 \%)$ in the splitting group and $1(5.0 \%)$ in the conventional group underwent revision surgery because of the slip progression. The cases of revision surgery are summarized in Table 3. 
Table 3. Summary of patients who underwent revision surgery

\begin{tabular}{lclc} 
Group & Period of revision surgery $(\mathrm{mo})$ & \multicolumn{1}{c}{ Cause of revision surgery } & Method of revision surgery \\
Splitting & 16 & Slip progression & PLIF \\
Splitting & 14 & Adjacent vertebral fracture & PLIF \\
\hline Splitting & 6 & Disc herniation & Herniotomy \\
Splitting & 4 & Radiculopathy at intervertebral foramen & PLIF \\
\hline Splitting & 17 & Disc herniation & Herniotomy \\
Splitting & 34 & Radiculopathy at intervertebral foramen & PLIF \\
Splitting & 28 & Radiculopathy at intervertebral foramen & PLIF \\
Conventional & 28 & Restenosis & Laminectomy \\
\hline Conventional & 18 & Slip progression & PLIF \\
\hline
\end{tabular}

PLIF, posterior lumbar interbody fusion.

\section{Discussion}

A commonly chosen surgical treatment for symptomatic LSS is direct cauda equina or nerve root decompression using a posterior approach. However, postoperative low back pain is a major problem in so-called failed back surgery syndrome. Previous investigators have reported that dissection of the paravertebral muscle can lead to denervation and atrophy, resulting in an increased risk of failed back surgery syndrome [1,7]. Histologic, enzymatic, and radiographic evidences of back muscle injury in lumbar surgery have been confirmed by several authors $[8,9]$. To solve these problems, various decompressive techniques for treating LSS have been developed with the intention of preserving the posterior supporting structures of the lumbar spine [3,10-14]. To avoid paravertebral muscle damage, we performed spinous process-splitting laminectomy, which was developed by Watanabe et al. [3] for patients with LSS. However, few studies have reported the clinical results of this procedure. We investigatedeffects of surgical invasion and clinical results, 2 years postoperatively using this procedure.

Muscle injury during surgical procedures causes an increase in CK level [15-17], and elevated CK activity after spinal surgery was reported in some of the studies [18-20]. In our study, mean CK level on the first postoperative day was higher in the conventional group than in the splitting group. However, there was no significant difference between the two groups $(p=0.161)$. Furthermore, mean CRP level on the first postoperative day was significantly higher in the conventional group than in the splitting group ( $p=0.006$ ). This finding may be due to less dam- age in the splitting group compared to the conventional group. The difference may become more significant if the number of cases in the conventional group is increased, because there was a great difference in the number of cases between the splitting ( 55 cases) and conventional (20 cases) groups. Furthermore, the mean number of days until returning to normal body temperature postoperatively was significantly lower in the splitting group than in the conventional group ( $p=0.047)$. These data suggest that the splitting procedure was less invasive.

JOA score improved significantly at 2 years postoperatively in both the splitting and conventional groups $(p<0.001$ and $p=0.021$, respectively). The recovery rate of JOA score in the splitting group was better than that in the conventional group, but there was no significant difference. Again, this difference may become more significant if the number of cases in the conventional group is increased. Therefore, spinous process-splitting laminectomy may be a better choice of procedure for treating LSS.

No study has investigated radiographic instability after spinous process-splitting laminectomy. In our study, radiographic evaluation showed that the slip progression was lower in the splitting group than in the conventional group. The mean change in angulation at 2 years postoperatively was significantly smaller in the splitting group than in the conventional group ( $p=0.007)$. Furthermore, the rate of revision surgery was similar in both groups; however, the rate of revision surgery due to slip progression was lower in the splitting group than in the conventional group (1.8\% vs. $5.0 \%)$. Spinous process-splitting laminectomy showed to be more stable according to 
radiographic evaluation. Although increases in translation $(0.71 \mathrm{~mm})$ and angulation $\left(3.5^{\circ}\right)$ were observed in the conventional group, they were not large enough to be judged as obvious instability.

The number of ASD in spinous process-splitting group was significantly lower than in conventional group, because the bone union and fibrous union were able to be achieved and the rate of ASD was reduced by preserving the posterior supporting structure. We consider that this contributed to our results where the progression of postoperative instability was lower in spinous processsplitting group, which consequently lowered the number of revision surgery due to slip progression in spinous process-splitting group.

This study has certain limitations, which includes a large difference in the number of patients between the two groups and having short terms for the follow-ups. However, the spinous process-splitting laminectomy achieved excellent clinical and radiographic results in this study.

\section{Conclusions}

Compared to the conventional laminectomy, the spinous process-splitting laminectomy may be less invasive and more stable for patients with LSS.

\section{Conflict of Interest}

No potential conflict of interest relevant to this article was reported.

\section{References}

1. Sihvonen T, Herno A, Paljarvi L, Airaksinen O, Partanen J, Tapaninaho A. Local denervation atrophy of paraspinal muscles in postoperative failed back syndrome. Spine (Phila Pa 1976) 1993;18:575-81.

2. Taylor H, McGregor AH, Medhi-Zadeh S, et al. The impact of self-retaining retractors on the paraspinal muscles during posterior spinal surgery. Spine (Phila Pa 1976) 2002;27:2758-62.

3. Watanabe K, Hosoya T, Shiraishi T, Matsumoto M, Chiba K, Toyama Y. Lumbar spinous process-splitting laminectomy for lumbar canal stenosis. Technical note. J Neurosurg Spine 2005;3:405-8.

4. Japanese Orthopaedic Association. Scoring system for low back pain. In: Japanese Orthopaedic Association, editor. Japanese Orthopaedic Association assessment criteria guidelines manual. Tokyo: Japanese Orthopaedic Association; 1996. p.46-9.

5. Hirabayashi K, Watanabe K, Wakano K, Suzuki N, Satomi K, Ishii Y. Expansive open-door laminoplasty for cervical spinal stenotic myelopathy. Spine (Phila Pa 1976) 1983;8:693-9.

6. Robertson JT, Papadopoulos SM, Traynelis VC. Assessment of adjacent-segment disease in patients treated with cervical fusion or arthroplasty: a prospective 2-year study. J Neurosurg Spine 2005;3:41723.

7. Weiner BK, Walker M, Brower RS, McCulloch JA. Microdecompression for lumbar spinal canal stenosis. Spine (Phila Pa 1976) 1999;24:2268-72.

8. Gejo R, Matsui H, Kawaguchi Y, Ishihara H, Tsuji H. Serial changes in trunk muscle performance after posterior lumbar surgery. Spine (Phila Pa 1976) 1999; 24:1023-8.

9. Kawaguchi Y, Matsui H, Tsuji H. Changes in serum creatine phosphokinase MM isoenzyme after lumbar spine surgery. Spine (Phila Pa 1976) 1997;22:1018-23.

10. Nakai O, Ookawa A, Yamaura I. Long-term roentgenographic and functional changes in patients who were treated with wide fenestration for central lumbar stenosis. J Bone Joint Surg Am 1991;73:1184-91.

11. O'Leary PF, McCance SE. Distraction laminoplasty for decompression of lumbar spinal stenosis. Clin Orthop Relat Res 2001;(384):26-34.

12. Poletti CE. Central lumbar stenosis caused by ligamentum flavum: unilateral laminotomy for bilateral ligamentectomy: preliminary report of two cases. Neurosurgery 1995;37:343-7.

13. Weiner BK, Fraser RD, Peterson M. Spinous process osteotomies to facilitate lumbar decompressive surgery. Spine (Phila Pa 1976) 1999;24:62-6.

14. Watanabe K, Matsumoto M, Ikegami T, et al. Reduced postoperative wound pain after lumbar spinous process-splitting laminectomy for lumbar canal stenosis: a randomized controlled study. J Neurosurg Spine 2011;14:51-8.

15. Nevins MA, Saran M, Bright M, Lyon LJ. Pitfalls in interpreting serum creatine phosphokinase activity. JAMA 1973;224:1382-7.

16. Penneys R, Wilkinson JH. Elevation of serum creatine kinase following amputation of the leg. Surgery 
1970;67:302-5.

17. Savignano T, Hanok A, Kuo J. Creatine phosphokinase activity. A study of normal and abnormal levels. Am J Clin Pathol 1969;51:76-85.

18. Lenke LG, Bridwell KH, Jaffe AS. Increase in creatine kinase $\mathrm{MB}$ isoenzyme levels after spinal surgery. J Spinal Disord 1994;7:70-6.

19. Healey JH, Kagen LJ, Velis KP, Levine DB. Creatine kinase MB in skeletal muscle and serum of spine-fusion patients. Clin Orthop Relat Res 1985;(195):2828.

20. Wukich DK, Van Dam BE, Graeber GM, Martyak T. Serum creatine kinase and lactate dehydrogenase changes after anterior approaches to the thoracic and lumbar spine. Spine (Phila Pa 1976) 1990;15:187-90. 